УДК 528.77

PACS 07.05.Pj

\title{
А. Н. Горобец
}

Харьковский национальный университет имени В. Н. Каразина

4, пл. Свободы, Харьков, 61022, Украина

E-mail: alex.n.gorobets@gmail.com

\section{ОБРАБОТКА МОНОХРОМНОГО ИЗОБРАЖЕНИЯ ПОВЕРХНОСТИ ЗЕМЛИ ДЛЯ ОБНАРУЖЕНИЯ СТРОЕНИЙ}

В статье представлен новый метод поэтапной обработки изображений для распознавания строений на черно-белых снимках дистанционного зондирования Земли в различных диапазонах длин волн.

Метод основан на особенностях формы большинства антропогенных объектов, границы которых представляют прямые линии, расположенные под прямым углом друг к другу. Представление изображения как раздельных массивов величины и направления градиента яркости позволяет обнаруживать объекты как в случае с белыми объектами на черном фоне, так и с черными - на белом. Разработанный двумерный адаптивный фильтр изображения позволяет обнаружить прямые края объекта, даже если фрагмент изображения имеет низкий контраст и высокий уровень шумов. Последующая обработка только списка отрезков, в отличие от обработки всего растрового изображения, позволяет существенно сократить время обработки.

Разработанный алгоритм при обработке исходного изображения формирует ограниченный набор объектов, форма которых близка к антропогенным.

Предложенный метод может быть использован в качестве алгоритма сегментации изображений и формирования на их основе гипотез для последующего распознавания при помощи нейронных сетей. Метод также продуктивен как средство автоматизации и повышения эффективности подготовки оператором обучающей выборки изображений для нейронной сети, а также оптимизации этого процесса. Ил. 5. Библиогр.: 13 назв.

Ключевые слова: космический землеобзор, обработка изображений, поиск прямых, адаптивный фильтр.

Современные технологии дистанционного зондирования Земли (ДЗ3) постоянно требуют разработки все более совершенных алгоритмов выявления антропогенных объектов на спутниковых снимках. Основные типы монохромных продуктов Д3З - радиолокационные изображения, снимки в инфракрасном диапазоне и оптические в панхроматическом режиме.

По ряду причин наиболее информативными и перспективными можно считать радиолокационные изображения (РЛИ), полученные радиолокаторами с синтезированной апертурой (РСА). Их особые свойства явились основой для разработки ряда методов решения задач, специфичных для РСА, основанных на изучении поля фрактальной размерности. К таким задачам относятся определение характеристик ледового покрова, картографирование океанических течений, поиск отмелей и др. Эти методы подробно рассмотрены в [1]. Однако применение поля фрактальной размерности для поиска зданий едва ли окажется достаточно эффективным в силу того, что предполагается выделять все здания на изображении вне зависимости от материала крыши и характера неоднородностей на ней. Таким образом, единственным общим критерием для всех зданий являются особенности формы контура.

C этой позиции наиболее удобными для начального этапа разработки алгоритма поиска антропогенных объектов являются снимки Земли в инфракрасном диапазоне. Объекты на таких изображениях не имеют теней, а уровень шума, как правило, относительно невелик. Сами же строения имеют контур, состоящий из прямых линий, которые попарно параллельны друг другу либо же пересекаются под прямым углом. Под подобное описание подпадает бо́льшая часть антропогенных объектов как таковых, поэтому целесообразно разработать алгоритм для определения координат в растре монохромного изображения всех возможных строений.

Таким образом, основная задача данной работы - программные решения для обнаружения на монохроматическом изображении строений (в англоязычной литературе - building extraction). Это направление представлено большим количеством работ, однако все соответствующие методы можно разделить на шесть вариантов: поиск граней на стереоизображениях [2]; определение специфических критериев, полученных из статистических свойств градиентов изображения объекта (HOG-дескрипторы [3]); определение критериев, полученных из особых точек (SIFT-дескрипторы [4]); коррекция гипотез, основанных на спектральных свойствах объектов $[5,6]$; обнаружение угловых точек с последующим уточнением контуров [7]; поиск прямых краев объекта и формирование гипотез на их основе $[8,9]$.

В самых современных и эффективных методах используется несколько описанных критериев с последующим взвешиванием [10], однако одиночное монохромное изображение не позволяет использовать большую их часть. Поскольку строения могут иметь различную форму, единственным критерием их достоверного выявления представляется наличие прямых границ и прямых углов между ними. Однако прямые границы, как правило, выглядят таковыми только визуально, на 
реальных космических снимках они зачастую представлены ломаными отрезками. Таким образом, практическая новизна данной работы сводится к более производительному алгоритму поиска прямых краев объектов, который более устойчив к неточностям контура.

В качестве исходных данных взята большая выборка инфракрасных снимков ДЗ3 [11], в которой каждый снимок имеет размер 16000 на 576 пикселей. Для верификации метода для каждого изображения использовались априорно известные растровые координаты одиночных зданий.

1. Адаптивный фильтр. После коррекции контраста и дифференцирования изображение передается в специальный фильтр. Разработанный автором двумерный адаптивный динамический фильтр выделяет прямые линии градиентов, а также линии градиентов с большим радиусом изгиба. На вход фильтра подаются два массива данных, равных по размеру входному изображению. Это массив значений величины градиента изображения $\mathbf{F}$ и массив направления градиента изображения $\boldsymbol{\theta}$ для каждого пикселя исходного изображения.

Адаптивный фильтр изображения был разработан в двух модификациях. Рассмотрим базовый вариант, работающий по следующей схеме. Для каждого пикселя независимо рассчитывается свое ядро свертки (фильтр), исходя из азимута градиента текущего пикселя. Например, в случае нулевого азимута ядро определяется как функция Гаусса для первого измерения и как константа для второго. Чтобы повернуть поверхность, описываемую двумерной функцией, на угол $\theta$ относительно начала координат, нужно выразить ее в полярной системе координат, вычесть требуемый угол и преобразовать обратно в декартову систему координат. Поскольку описанная выше функция зависит только от одного измерения, удобно вынести сочетание якобианов в отдельное выражение для «подставных» координат:

$\mathbf{x}_{i, j}=\sqrt{(c-i)^{2}+(c-j)^{2}} \cos \left[\operatorname{arctg}\left(\frac{c-i}{c-j}\right)-\theta\right]$,

где $\mathbf{x}_{i, j}-$ значения координаты $X$ на повернутой

на угол $\theta$ координатной плоскости в точках, соответствующих целым значениям положений строки $i$ и столбца $j$ в ядре фильтра; $c=(s+1) / 2-$ адрес центра ядра (как по строкам, так и по столбцам); $s$ - размер ядра.

Для поворота используются непосредственно значения направлений градиентов $\boldsymbol{\theta}_{\text {row,col }}$, поскольку при нулевом повороте (градиент изображения направлен вправо) измерение $X$ (номер столбца) имеет форму гауссовой функции, а вдоль $Y$ расположена линия максимальных значений. Так, линия максимальных значений ядра приходится на соседние (выше и ниже) пиксели изображения, которые лежат на текущей линии градиента (перпендикулярно направлению градиента в текущем пикселе). Следовательно, если текущий пиксель является частью прямой линии градиента изображения, т. е. контура объекта, то максимальные значения ядра соответствуют пикселям, которые имеют одинаковое направление градиента и, возможно, принадлежат той же линии градиента. Это свойство сохраняется при любом повороте ядра. Базовый вариант ядра адаптивного фильтра определен выражением:

$$
\mathbf{B}_{i, j}=\frac{1}{\sigma \sqrt{2 \pi}} \exp \left(-\frac{\mathbf{x}_{i, j}{ }^{2}}{2 \sigma^{2}}\right),
$$

где $\sigma$ - параметр гауссианы.

На рис. 1, а представлены фрагмент исходного изображения и соответствующее центральному пикселю фрагмента базовое ядро размером $11 \times 11$ пикселей с $\sigma$-параметром, равным единице. Из рисунка видно, что такой динамический фильтр будет наиболее эффективно подсвечивать прямые границы объектов либо же границы с достаточно большим радиусом изгиба.

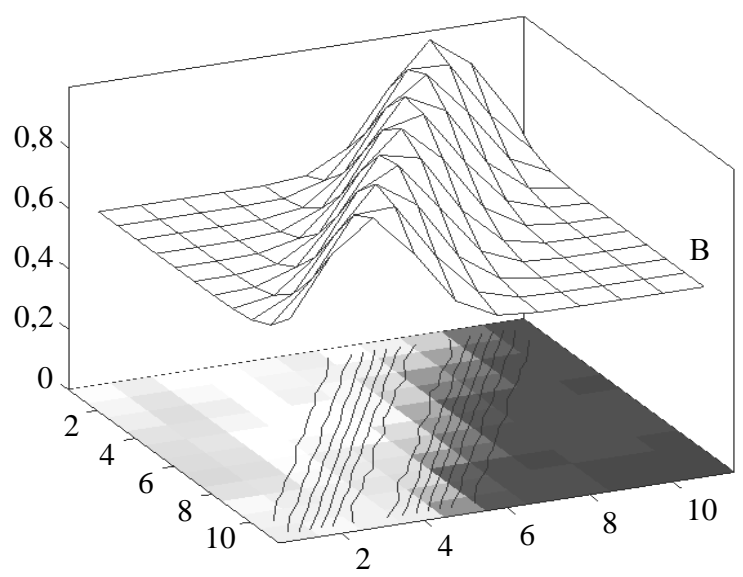

a)

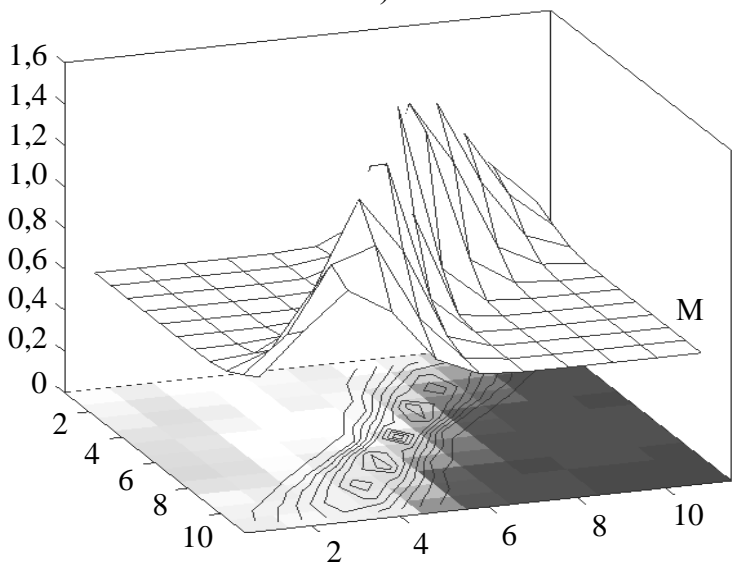

б)

Рис. 1. Фрагмент изображения прямой линии градиента и соответствующие центральному пикселю базовое ядро адаптивного фильтра (а) и модифицированное ядро (б) 
Для уменьшения влияния флуктуаций азимута на изображении было предложено модифицированное ядро, элементы которого вычисляются в соответствии с выражением:

$$
\mathbf{M}_{i, j}=\cos \left(\frac{2 R}{s}\right) \exp \left(-\frac{\mathbf{x}_{i, j}{ }^{2}}{2\left(\sigma+R K_{\sigma}\right)^{2}}\right),
$$

где $K_{\sigma}-$ коэффициент роста параметра $\sigma ; s-$ размер ядра; $R$ - расстояние от каждого элемента до центра ядра (определяется на этапе вычисления $\left.\mathbf{x}_{i, j}\right)$ :

$$
R=\sqrt{(c-i)^{2}+(c-j)^{2}} .
$$

На рис. 1, б виден конусообразный спад весов на краях модифицированного ядра адаптивного фильтра. Также в модели ядра используется коэффициент, увеличивающий местный $\sigma$-параметр по мере удаления от центра ядра $K_{\sigma}$. Данные преобразования позволили добиться желаемого понижения чувствительности фильтра к флуктуациям азимута градиента, а также сделать его более чувствительным к резким перепадам яркости.

Далее рассмотрим подробнее сам процесс фильтрации с полученным ядром. В нашем случае входных массивов два: массив направлений $\boldsymbol{\theta}$ и модулей градиентов $\mathbf{F}$. Для получения конечного результата фильтрации по каждому пикселю используется массив модуля градиента, в то время как массив направления используется для вычисления разницы направления градиента между текущим пикселем и каждым из окрестных пикселей, которые находятся в пределах текущего положения окна фильтра:

$$
\text { AxDiff }_{i, j}=\left|\boldsymbol{\theta}_{\text {row }+i-c, c o l+j-c}-\boldsymbol{\theta}_{\text {row }, c o l}\right|,
$$

где row, col - номера строки и столбца текущего пикселя, для которого ищется очередное выходное значение фильтра, соответственно; $i, j \in[1, s]$ - итераторы строк и столбцов элементов подмножества разности направлений градиентов для текущего положения ядра; $c=(s+1) / 2-$ адрес центра ядра в его локальных координатах; $s$ - размер ядра.

При определении конечного значения текущего пикселя изображения-результата адаптивного фильтра можно сказать, что он обратно пропорционален полученным разностям направлений градиентов (с учетом весов, определяемых ядром фильтра), но прямо пропорционален величинам пиксельных градиентов. Таким образом, исключающее возможность деления на ноль выражение имеет такую форму:

$$
\mathbf{A d F}_{r o w, c o l}=\sum_{i=1}^{s} \sum_{j=1}^{s} \mathbf{M}_{i, j} \frac{\mathbf{F}_{r o w+i-c, c o l+j-c}}{1+W \mathbf{A d D i f f}_{i, j}},
$$

где $W$ - обобщенный вес азимутальной составляющей (при $W=0$ учитываются только величины градиентов). Вся дробь не может принять значения выше градиента $\mathbf{F}$ (в текущем пикселе), а размер «окрестностей» по уровню половины от максимального градиента всегда равен $W$.

Результат работы фильтра по данному соотношению представлен на рис. 2, б; его можно сравнить с соответствующим массивом величины градиента $\mathbf{F}$ (рис. 2, а).

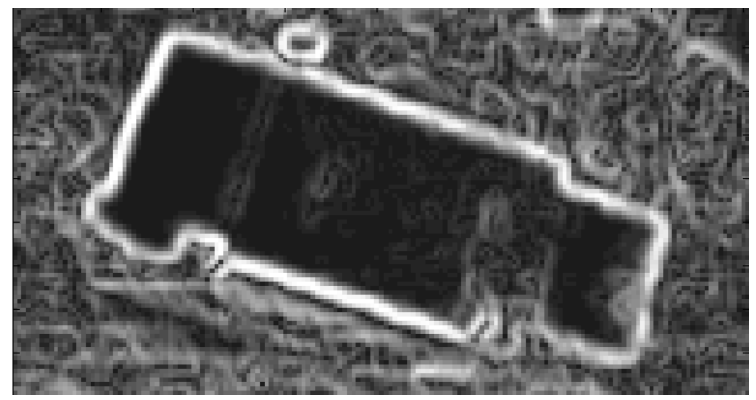

a)

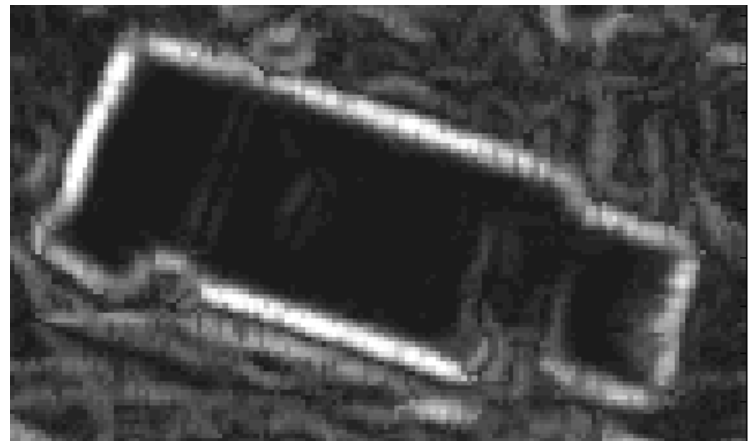

б)

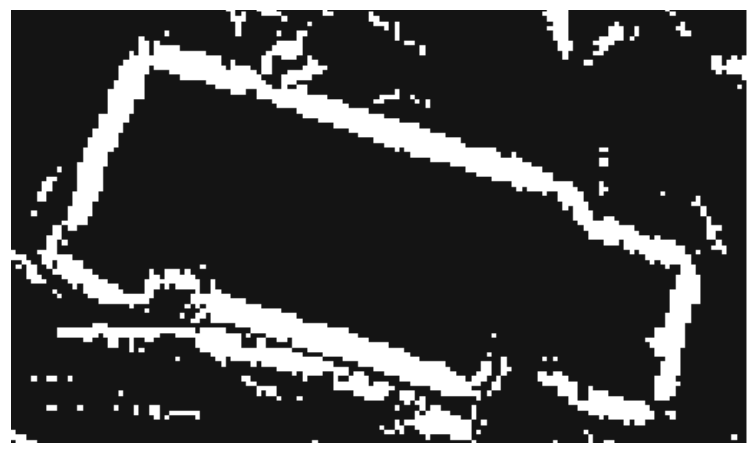

B)

Рис. 2. Массив $\mathbf{F}$ - абсолютные значения градиента тестового изображения (a), результат параметризованной адаптивной фильтрации (б) и полученная бит-маска (в)

На рис. 2, б плавные градиенты переданы толстыми линиями, что и требуется для дальнейшей обработки, поскольку на следующем этапе формирования отрезков контура потребуется максимально доступное количество пикселей, которые отвечают за каждый из прямолинейных градиентов (область прямолинейного градиента). 
2. Определение линейных фрагментов контура. Рассмотрим результат адаптивной фильтрации (рис. 2, б). Вычислив среднее значение яркости такого изображения, легко получить бинарную маску, где светлые пиксели соответствуют пикселям - участникам формирования отрезков на исходном изображении (рис. 2 , в).

Далее необходимо полученные группы пикселей, которые соответствуют целостному объекту, разделить на подгруппы, каждая из которых соответствует своему отрезку. Чтобы реализовать программное решение, устойчивое к изменениям шумов и масштаба, необходимо разработать функцию, которая возвращала бы список отрезков как наиболее общих элементов контура, так и его мелких деталей. Следовательно, в случаях, когда из одних и тех же пикселей может получиться как одна длинная линия, так и несколько коротких, алгоритм должен возвращать все возможные линии, предоставляя таким образом избыточную информацию о контуре (и обобщенную, и детальную).

Это удалось реализовать путем итерационного изменения диапазонов азимута, по которым выбираются пиксели для отрезков текущей итерации (рис. 3). На рис. 3 показаны бит-маски с текущими азимутами градиентов изображения, которые соответствуют четырем сторонам объекта. Для вычисления этих бит-масок использован слой разницы азимутов значений из массива $\boldsymbol{\theta}$ и азимута текущей итерации, который определен выражением:

AdDiffLayer $_{r o w, c o l}=\left|\boldsymbol{\theta}_{\text {row }, c o l}-\left(-\pi+t / N_{\text {Step }} 2 \pi\right)\right|$,

где $\boldsymbol{\theta}$ - массив направлений (азимутов) градиента изображения; $t$ - номер итерации; $N_{S t e p}-$ количество итераций направления градиента в цикле. Здесь азимут итерации определен в радианах в пределах от $-\pi$ до $\pi$.

В каждой итерации из полученного массива разности азимутов градиентов вычисляется текущий слой значимости пикселей:

$$
\begin{aligned}
& \text { CrntLayer }_{r o w, c o l}= \\
& =\frac{\mathbf{A d F}_{r o w, c o l}}{\mathbf{A d D i f f L a y e r}_{r o w, c o l} /\left(F_{O v} 2 \pi / N_{\text {Step }}\right)},
\end{aligned}
$$

где $F_{O v}$ - коэффициент перекрытия диапазонов азимута. Поскольку AzdiffLayer хранит фактические разности азимутов градиента изображения для каждого пикселя и азимута текущей итерации в радианах, результат динамической фильтрации делится на эту разность. Так обеспечивается участие пикселей с бо́льшим значением в $\mathbf{A d F}$ в бо́льшем количестве итераций азимута. Поскольку разница азимутов в один радиан очевидно слишком велика, AzdiffLayer, в свою очередь, нормирован на меньшую величину - в данном случае, сектор (ширину диапазона углов) одной итерации $\left(2 \pi / N_{\text {Step }}\right)$.

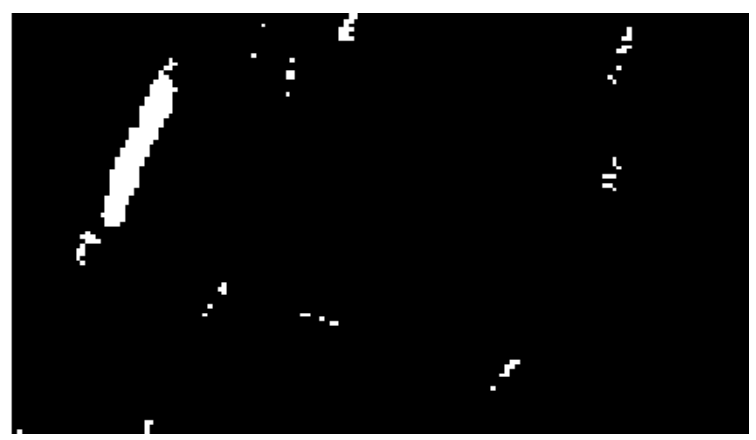

a)

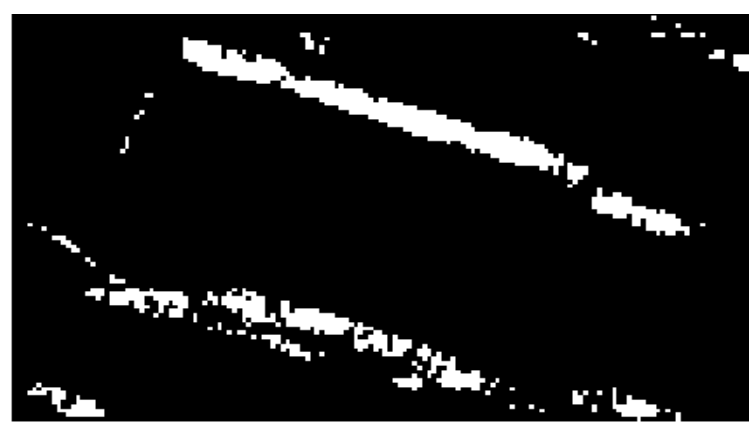

б)

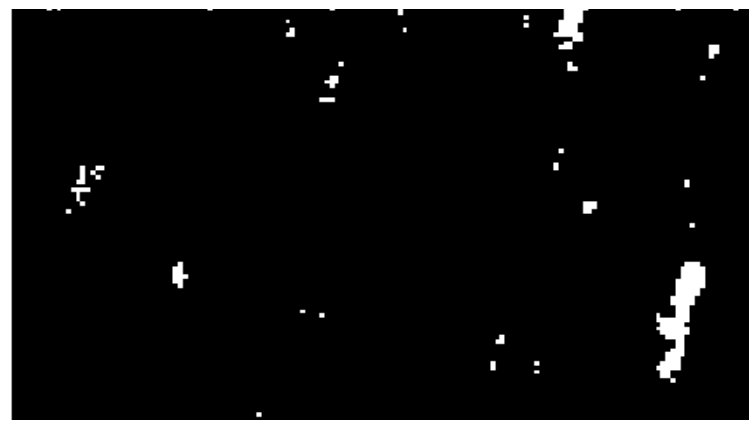

в)

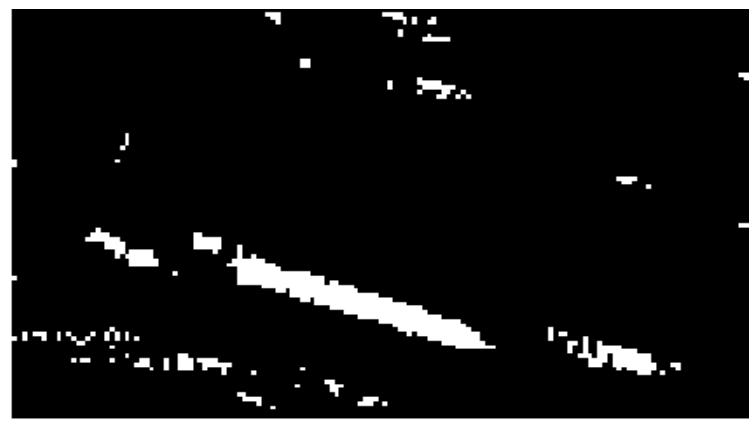

г)

Рис. 3. Бинарные маски для опорных азимутов, соответствующих сторонам объекта: а) $160^{\circ}$; б) $68^{\circ}$; в) $12^{\circ}$; г) $104^{\circ}$

С другой стороны, в зависимости от уровня флуктуаций азимута градиента, может оказаться, что пиксели от одной прямой кромки попадут в разные итерации. Для решения этой проблемы 
введен коэффициент перекрытия $F_{O v}$, который задан в долях сектора одной итерации.

Полученное изображение подвергается бинаризации с тем же порогом, что и основная битмаска (рис. 3). Поскольку околонулевая разница азимута в массиве AzdiffLayer нередко формирует связные области (островки) с заведомо низким градиентом, дополнительно используется основная бит-маска, полученная непосредственно из AdF. Соответственно, выражение для текущей бит-маски в каждой итерации следующее:

CrntBM $_{i, j}=\left(\right.$ CrntLayer $\left._{i, j}>T\right) \wedge$ MainBM $_{i, j}$,

где $T$ - порог бинаризации; МаinBM - основная бит-маска (рис. 2, в).

Таким образом, по мере вращения опорного азимута в каждой итерации сначала проявляются пиксели с наибольшим градиентом в отдельных островках, что соответствует высокой детализации (мелкие подробные отрезки), затем (когда опорный азимут наиболее соответствует ориентации кромки объекта) вся линия выделяется в один островок, что соответствует максимальному обобщению. Таким образом реализован принцип избыточности информации о контуре, при этом условный предел «уровня обобщения» определяется $\sigma$-параметром динамического фильтра и коэффициентом перекрытия $F_{O v}$. Эти же параметры определяют устойчивость алгоритма к флуктуациям азимута градиента (шумам на изображении).

3. Формирование списка отрезков. Из полученных связных областей (островков) далее необходимо получить вписанные отрезки в виде списка их параметров. Главным образом, это координаты краев отрезка $\left(X_{1,2}\right.$ и $\left.Y_{1,2}\right)$. Также в качестве вспомогательных данных указываются координаты центральной точки отрезка $\left(X_{C P}, Y_{C P}\right)$ и направление градиента изображения, описываемого этой линией $\left(G_{A z}\right)$, который по сути является векторной суммой градиентов от каждого пикселя в группе. Кроме того, в качестве параметров достоверности для каждого отрезка указаны количество пикселей в группе (островке) и параметр разброса азимутов пиксельных градиентов в текущей группе $\left(D_{A z}\right)$.

Прежде всего текущая группа связных пикселей проверяется на достаточность количества пикселей. Это минимальное допустимое количество равно $1 / \sqrt{2} L_{\min }$ ( $L_{\min }$ - минимальная длина отрезка, выбранная пользователем). Затем, определяются параметры оптимальной прямой вписанной в «островок» пикселей согласно следующей модели:

$$
y=\operatorname{tg}\left(G_{A z}+\frac{\pi}{2}\right)\left(x-X_{C P}\right)+Y_{C P},
$$

$$
G_{A z}=\operatorname{arctg}\left(\frac{\sum_{t \in \operatorname{str} N} d x_{t}}{\sum_{t \in \operatorname{str} N} d y_{t}}\right),
$$

где $G_{A z}$ - средневзвешенное направление градиента в текущей группе пикселей, найденное путем суммирования производных по каждому пикселю из группы, которые были найдены на этапе вычисления массивов величины $\mathbf{F}$ и направления $\boldsymbol{\theta}$ градиентов изображения.

Введем параметр $t=\mathbf{c o l}_{t} N_{\text {row }}+$ row $_{t}-$ разыменованный (одномерный) индекс для обращения к множеству связных пикселей текущей группы (островка), где $\mathbf{c o l}_{t}, \mathbf{r o w}_{t}-$ список, содержащий порядковые номера столбца и строки $t$-го (текущего) пикселя из данного островка соответственно; $N_{\text {row }}$ - количество строк в изображении (растре). Теперь для обращения к каждому пикселю из островка требуется уже не два списка (номеров строк и соответствующих им номеров столбцов по каждому пикселю из группы), а один список порядковых номеров пикселей в растре. Обозначим $\operatorname{str} \mathbf{N}$ - множество индексов $t$, соответствующих текущей группе связных пикселей. Тогда $X_{C P}, Y_{C P}-$ абсцисса и ордината средневзвешенного центра отрезка - выражаются как:

$X_{C P}=\frac{\sum_{t \in \mathbf{s t r N}} \operatorname{col}_{t} \mathbf{F}_{t}}{\sum_{t \in \mathbf{s t r} \mathbf{N}} \mathbf{F}_{t}}, \quad Y_{C P}=\frac{\sum_{t \in \mathbf{s t r N}} \operatorname{row}_{t} \mathbf{F}_{t}}{\sum_{t \in \mathbf{s t r} \mathbf{N}} \mathbf{F}_{t}}$.

В целом, модель (2) является модификацией простейшего уравнения $y=k x+b$, поэтому для того чтобы вычислить координаты краев отрезка, необходимо сначала определить границы текущей группы («фрейма»):

$$
\begin{array}{ll}
P_{L f t}=\min \left(\text { col }_{t}\right), & P_{R g h t}=\max \left(\operatorname{col}_{t}\right), \\
P_{U p}=\min \left(\text { row }_{t}\right), & P_{D w n}=\max \left(\text { row }_{t}\right),
\end{array}
$$

Для определения точек пересечения найденной прямой с левой и правой границами фрейма необходимо подставить $P_{L f t}$ и $P_{R g h t}$ вместо $x$ в (2). Полученные значения обозначим $Y_{L f t}$ и $Y_{R g h t}$ соответственно. Для горизонтальных границ можно выразить это уравнение относительно $y$ :

$$
x=\frac{y-Y_{C P}}{\operatorname{tg}\left(G_{A z}+\frac{\pi}{2}\right)}+X_{C P} .
$$

Для поиска точек пересечения уравнения прямой и верхней (нижней) границ фрейма необходимо в данное уравнение аналогично подставить $P_{U p}$ и $P_{D w n}$ вместо $y$, чтобы получить $X_{U p}$ и $X_{D w n}$. Все основные элементы при вычислении параметров вписанного в островок отрезка показаны на рис. 4 .

Из полученных четырех точек пересечения выбираются те, которые лежат в пределах отрезков границ фрейма. Искомая пара точек должна 
подчинятся таким правилам для вертикальных и горизонтальных границ фрейма соответственно:

$P_{R g h t}>X_{U p, D w n}>P_{L f t}$,

$P_{D w n}>Y_{L f t, R g h t}>P_{U p}$.

Подразумевается, что ось $Y$ направлена вниз (координата растет вместе с ростом адреса строки row в растре), поэтому численно $P_{D w n}>P_{U p}$.

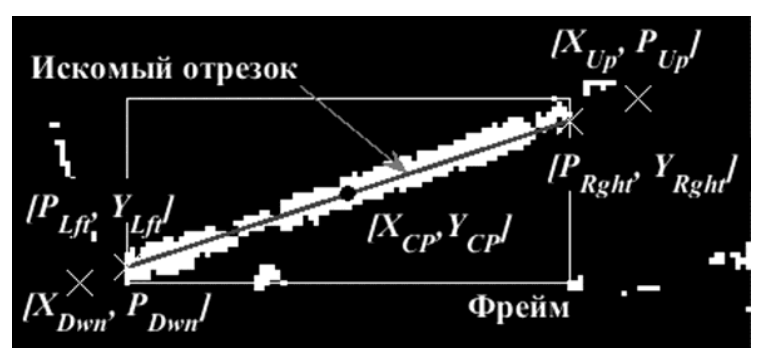

Рис. 4. Пример формирования параметров отрезка

Ключевым параметром значимости для каждого отрезка является параметр разброса азимутов градиентов по множеству пикселей данной группы:

$$
D_{A z}=\frac{G_{A z}}{\sum_{t \in \mathbf{s t r N}} \mathbf{F}_{t}} \text {. }
$$

В результате получена величина, указывающая на разброс азимутов, которая изначально нормирована (не превышает единицу) и не зависит от количества пикселей. Таким образом, формируется список параметров, достаточный для построения этих отрезков поверх растра.

4. Выявление объектов с формой, характерной для строений. Как было отмечено выше, в качестве основных признаков антропогенного объекта на изображении были приняты наличие прямых границ объекта и прямых углов между ними. В качестве первого шага необходимо объединить отрезки в объекты-углы.

Для того чтобы избавиться от необходимости сопоставлять каждый отрезок со всеми остальными, удобно сначала отсортировать весь список по ориентации отрезков, чтобы потом разделить его на два списка - так называемые Master- и Slave-отрезки. Каждый отрезок из первого списка сопоставляется только с теми Slave-отрезками, которые входят в требуемый диапазон по азимуту (ориентации). Иначе говоря, в первом списке присутствуют все отрезки с ориентацией от $-\pi$ до $0+T_{\text {RghtAng, }}$, а во втором от $0-T_{\text {RghtAng }}$ до $\pi$, где $T_{\text {RghtAng }}$ - устанавливаемый пользователем допуск, по которому угол можно считать прямым (в данной работе $\left.\pm 15^{\circ}\right)$. Далее по каждому Masterотрезку ведется поиск первого подходящего по азимуту Slave-отрезка, а сопоставление происхо- дит в цикле начиная с этого адреса и до первого Slave-отрезка с азимутом (ориентацией), превышающим заданный предел. «Прямым углом» считаются отрезки, сориентированные под близким к прямому углом друг к другу и расположенные достаточно близко к взаимной точке пересечения. Если эта точка лежит за пределами обоих отрезков, то расстояние от точки пересечения до ближайшего края каждого отрезка должно быть не более $L_{\text {CornMinDist }}$ (для определенности выберем 9 пикселей). Таким образом, по завершении процедуры формирования объектов-углов формируется бит-маска размером в длину Master-списка на длину Slave-списка, где каждая единица соответствует своему объекту-углу (паре отрезков).

Конечной целью данной работы является выделение потенциальных объектов-строений. Определим, что изображение искомого объекта (строения) должно иметь, по крайней мере, три прямых стороны и, соответственно, два прямых угла между ними. В контексте бит-маски соотношений Master- и Slave-отрезков это означает, что две единицы (соответствующих объектам-углам) должны находиться в одной строке, если у них общий Master-отрезок, или в одном столбце, если у них общий Slave-отрезок. В действительности объекты могут быть выражены и более сложными связями, поэтому была разработана специальная процедура рекурсивного поиска связей (строчных и столбцовых) в бит-маске. Такое решение может выявлять объекты, состоящие из неограниченного количества линий. Пример выявления рассматриваемого объекта приведен на рис. 5.

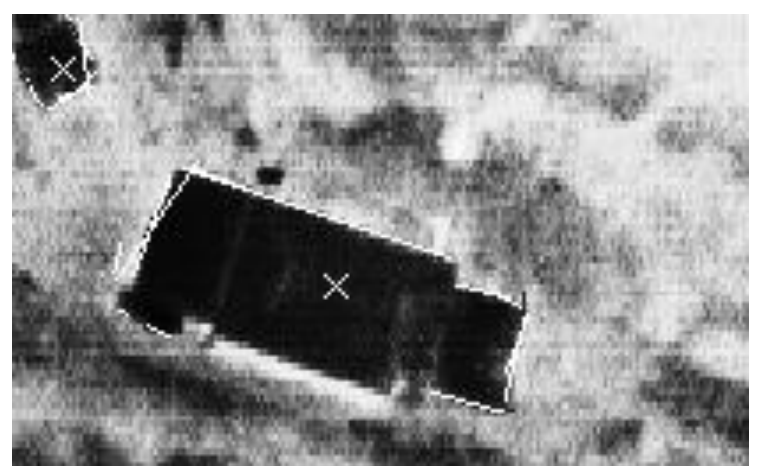

Рис. 5. Отрезки, сведенные в объект-строение

Из рисунка видно, что объект-строение состоит из объектов-углов с отрезками длиной более десяти пикселей. Более короткие отрезки не рассматривались в силу ограничений доступных вычислительных ресурсов.

В текущей реализации разработанный алгоритм является удобным инструментом, который выявляет практически все антропогенные объекты или, иначе говоря, объекты неестественной 
формы. С его помощью оператор может не извлекать самостоятельно объекты для каждого класса, а просто отсортировать предложенные объекты по классам. Этот алгоритм позволяет быстро получить обширный альбом изображений зданий, мостов, железнодорожных переездов, а также альбом контрастных объектов редкой формы, которые однако строениями не являются.

5. Оценка производительности метода. По результатам испытаний на 20-ти изображениях было выявлено 1836 потенциальных антропогенных объектов. При этом по итогам разметки вручную на этих снимках были отмечены 253 здания, из которых только 4 не были выделены алгоритмом.

Что касается альтернативных решений, то исходные наборы данных по опубликованным работам других авторов [2-7, 9, 10], как правило, в открытом доступе отсутствуют либо представлены снимками других типов. Можно сравнить эффективность выявления прямых фрагментов контура на одном наборе данных при помощи преобразования Хаффа [12] и с использованием разработанных автором программных решений. Данное преобразование применяется к бит-маскам с отмеченным контуром толщиной в один пиксель, поэтому на вход преобразования подаются не выделенные ранее области прямолинейного градиента, а контуры, полученные по методу Канни [13]. Результаты такой обработки можно видеть на рис. 6, а. В сравнении с разработанным алгоритмом (рис. 6, б) условно прямые фрагменты контуров по Хаффу выделяются хуже (особенно те из них, фрагменты которых наиболее отклоняются от прямолинейности).

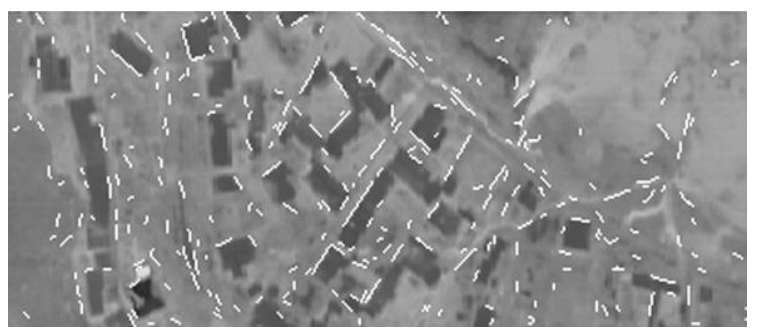

a)

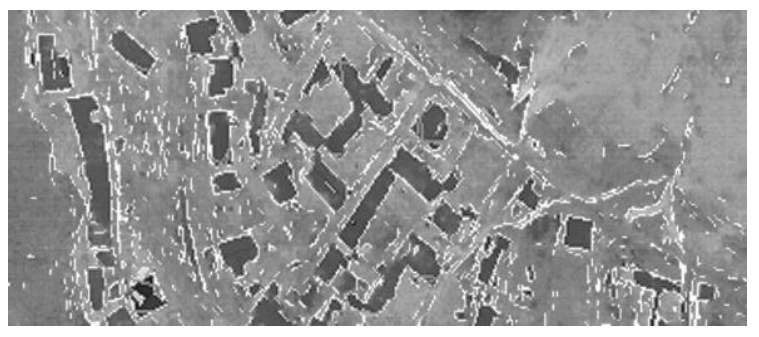

б)

Рис. 6. Линии прямых фрагментов контуров, полученные при помощи преобразования Хаффа (а) и построенные с помощью алгоритма (б)
Кроме того, короткие границы (порядка десяти пикселей) по методу Хаффа, как правило, не выделены. Очевидная причина такого результата подача на вход детектора границ Канни размытого (по Гауссу, $\sigma=2,5)$ изображения. Само же «размытие» обеспечивает подавление шумов и лучшее извлечение длинных условно прямых краев. При этом для изображений с такими мелкими объектами, очевидно, преобразование Хаффа плохо применимо, его несомненным преимуществом является высокая скорость обработки на каждое изображение требуется менее 15 секунд.

Выводы. Таким образом, к специфическим преимуществам разработанного алгоритма можно отнести:

- простоту (обобщенность) критериев выбора контура - только наличие прямых линий и прямых углов;

- устойчивость к шумам и искажениям формы контура;

- отсутствие квантования по ориентации искомых краев;

- стабильность работы алгоритма вследствие слабой чувствительности к контрастности изображения и нечувствительности к количеству искомых объектов на одном снимке.

К недостаткам предложенного алгоритма можно отнести относительно большое время обработки. Однако его можно существенно уменьшить, раскрыв потенциал алгоритма по использованию ресурсов видеоадаптера и используя более современные вычислительные мощности, которые совершенствуются с каждым годом.

\section{Библиографический список}

1. Фрактальный анализ процессов, структур и сигналов. Под ред. Р. Э. Пащенко. Харьков: Эко-Перспектива, 2006. $347 \mathrm{c}$.

2. Woo D.-M., Nguyen Q.-D., Nguyen Tran Q.-D., Park D.-C., Jung Y.-K. Building Detection and Reconstruction from Aerial Images. Proc. Int. Soc. for Photogrammetry and Remote Sensing (ISPRS). Beijing, China, July 3-11, 2008.

3. Konstantinidis D., Stathaki T., Argyriou V. and Grammalidis N. A probabilistic feature fusion for building detection in satellite images. Proc. $10^{\text {th }}$ Int. Conf. on Computer Vision Theory and Applications (VISAPP 2015). Vol. 2. Berlin, Germany, 11-14 March, 2015. SciTePress, pp. 205-212. DOI: 10.5220/0005260502050212

4. Sirmacek B. and Unsalan C. Urban-area and building detection using SIFT keypoints and graph theory. IEEE Trans. Geosci. Remote Sens. 2009. Vol. 47, N 4. P. 1156-1167.

5. Singh D., Maurya R., Shukla A. S., Sharma M. K. and Gupta P. R. Building extraction from very high resolution multispectral images using NDVI based segmentation and morpholo-gical operators. 2012 Students Conference on Engineering and Systems (SCES). Allahabad, Uttar Pradesh, India, 16-18 March 2012, pp. 1-5.

6. Hermosilla T., Ruiz L., Recio J., and Estornell J. Evaluation of automatic building detection approaches combining high resolution images and LiDAR data. Remote Sens. 2011. Vol. 3, N 6. P. 1188-1210. DOI:10.3390/rs3061188

7. Theng L. B. Automatic building extraction from satellite imagery. Engineering Letters. 2006. Vol. 13, N 3. [pdf] Eng. 
Lett., 13(3), 5 p. Aviable at: http://www.engineeringletters.com/ issues_v13/issue_3/EL_13_3_5.pdf.

8. Kahn P., Kitchen L., Riseman E. Fast Line Finder for VisionGuided Robot Navigation. IEEE Trans. Pattern Anal. Mach. Intell. 1990. Vol. 12, N 11. P. 1098-1102. DOI: 10.1109/ 34.61710

9. Haverkamp D. Automatic building extraction from IKONOS imagery. Proc. ASPRS Annual Conf. Denver, CO, USA, 23-28 May 2004.

10. Konstantinidis D., Stathaki T., Argyriou V., Grammalidis N. Building Detection Using Enhanced HOG-LBP Features and Region Refinement Processes. IEEE J. Sel. Top. Appl. Earth Obs. Remote Sens. 2017. Vol. 10, N 3. P. 888-905. DOI: 10.1109/RAST.2013.6581176

11. Конкурсы анализа данных [on-line]. URL: http://dataring.ru/ competitions/fpi-object-detection/

12. Method and means for recognizing complex patterns: Pat. 3,069,654 U. S. / P. Hough. Dec. 1962.

13. Canny J. A Computational Approach to Edge Detection. IEEE Trans. Pattern Anal. Mach. Intell. 1986. Vol. PAMI-8, N 6. DOI: 10.1109/TPAMI.1986.4767851

\section{REFERENCES}

1. Pashchenko, R. E. ed., 2006. Fractal analysis of processes, structures and signals. Kharkiv: EcoPerspectiva Publ. (in Russian).

2. Woo, D.-M., Nguyen, Q.-D., Nguyen Tran, Q.-D., Park, D.-C., Jung, Y.-K., 2006. Building detection and reconstruction from aerial images. In: Proc. Int. Soc. for Photogrammetry and Remote Sensing (ISPRS). Beijing, China, July 3-11, 2008.

3. Konstantinidis, D., Stathaki, T., Argyriou, V. and Grammalidis, N., 2015. A probabilistic feature fusion for building detection in satellite images. In: Proc. $10^{\text {th }}$ Int. Conf. on Computer Vision Theory and Applications (VISAPP 2015). Vol. 2. Berlin, Germany, 11-14 March, 2015, pp. 205-212. SciTePress. DOI: 10.5220/0005260502050212

4. Sirmacek, B. and Unsalan, C., 2009. Urban-area and building detection using SIFT keypoints and graph theory. IEEE Trans. Geosci. Remote Sens., 47(4), pp. 1156-1167.

5. Singh, D., Maurya, R., Shukla, A., Sharma, M. and Gupta, P., 2012. Building extraction from very high resolution multispectral images using NDVI based segmentation and morphological operators. In: 2012 Students Conf. Engineering and Systems. Allahabad, Uttar Pradesh, India, 16-18 March 2012, pp. 1-5.

6. Hermosilla, T., Ruiz, L., Recio, J. and Estornell, J., 2011. Evaluation of automatic building detection approaches combining high resolution images and LiDAR data. Remote Sens., 3(6), pp. 1188-1210. DOI:10.3390/rs3061188

7. Theng L. B., 2006. Automatic building extraction from satellite imagery. [pdf] Eng. Lett., 13(3), 5 p. Aviable at: http://www.engineeringletters.com/issues_v13/issue_3/EL_13 _3_5.pdf.

8. Kahn, P., Kitchen, L., Riseman, E., 1990. Fast Line Finder for Vision-Guided Robot Navigation. IEEE Trans. Pattern Anal. Mach. Intell., 12(11), pp. 1098-1102. DOI: 10.1109/34.61710

9. Haverkamp, D., 2004. Automatic building extraction from IKONOS imagery. In: Proc. ASPRS Annual Conf. Denver, CO, USA, 23-28 May 2004.

10. Konstantinidis, D., Stathaki, T., Argyriou, V., Grammalidis, N., 2017. Building Detection Using Enhanced HOG-LBP Features and Region Refinement Processes. IEEE J. Sel. Top. Appl. Earth Obs. Remote Sens., 10(3), pp. 888-905. DOI: 10.1109/RAST.2013.6581176

11. Competitions data analysis [on-line]. Available at: http://dataring.ru/competitions/fpi-object-detection/ (in Russian).

12. Hough, P., 1962. Method and means for recognizing complex patterns. U. S. Pat. 3,069,654.
13. Canny, J., 1986. A Computational Approach to Edge Detection. IEEE Trans. Pattern Anal. Mach. Intell., PAMI-8(6), pp. 679-698. DOI: 10.1109/TPAMI.1986.4767851

Рукопись поступила 18.10.2017.

$$
\text { A. N. Gorobets }
$$

\section{PROCESSING A MONOCHROMATIC EARTH OBSERVATION IMAGE FOR BUILDINGS DETECTION}

The purpose of this work is to develop a new technique of image processing, in particular for buildings detection on monochromatic Earth-observation images of different types.

The method is based on the property of most man-made objects which consists in straight edges and mostly right angles. Representing an image separately as gradient value- and directionlayers allows to detect targets in both cases: a bright object on a dark background and a dark one on a light background. The developed 2D adaptive image filter allows to detect straight edges even if the given image fragment has a low contrast and is extremely noised. The next processing of line-segment list without image raster works faster and allows to detect a small set of possible targets.

The developed algorithm forms a limited set of objects which shape is close to anthropogenic by processing the original image.

The proposed method can be used as an image segmentation algorithm for formation of hypotheses and subsequent recognition using neural networks. The method is also useful as a means for automating and improving the efficiency of training dataset images preparing by an operator for a neural network, and thereby as optimizing this process.

Key words: Earth observation, image processing, straight lines finding, adaptive filter.

\section{О. М. Горобець}

\section{ОБРОБЛЕННЯ МОНОХРОМНОГО ЗОБРАЖЕННЯ ЗЕМНОЇ ПОВЕРХНІ ДЛЯ ВИЯВЛЕННЯ СПОРУД}

У статті описано новий метод поетапної обробки зображень для виявлення будівель на чорно-білих знімках дистанційного зондування Землі в різних діапазонах довжин хвиль.

Метод заснований на особливостях форми більшості антропогенних об'єктів, контури яких складаються 3 прямих країв, розташованих під прямим кутом один до одного. Представлення зображення у вигляді роздільних масивів величини і напрямку градієнта яскравості дозволяє виявляти об'єкти як у випадку з білими об'єктами на чорному тлі, так і з чорними - на білому. Розроблений двовимірний адаптивний фільтр зображення дозволяє виявити прямі краї об'єкта навіть якщо фрагмент зображення має низький контраст і високий рівень шумів. Подальша обробка тільки списку відрізків, на відміну від оброблення всього растрового зображення, дозволяє істотно скоротити час обробки.

Розроблено алгоритм, який при обробці вихідного зображення формує обмежений набір об'єктів, форма яких близька до антропогенних.

Запропонований метод може бути використаний в якості алгоритму сегментації зображень і формування на їх основі гіпотез для подальшого розпізнавання за допомогою нейронних мереж. Метод також продуктивний як засіб для автоматизації та підвищення ефективності підготовки оператором тренувальної вибірки зображень для нейронної мережі, а отже, для оптимізації цього процесу.

Ключові слова: космічний землеогляд, оброблення зображень, пошук прямих, адаптивний фільтр. 